\title{
Existe associação entre a eletroneuromiografia e a ultrassonografia no diagnóstico da Síndrome do Túnel do Carpo?*
}

\section{Is There an Association between Electroneuromyography and Ultrasound in the Diagnosis of Carpal Tunnel Syndrome?}

\author{
Henver Ribeiro Paiva Filho ${ }^{10}$ Bruno Adriano Borges Elias ${ }^{10}$ Marlus Sérgio Borges Salomão Junior ${ }^{10}$

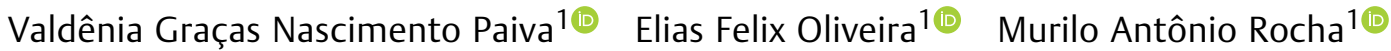 \\ 1 Serviço de Cirurgia da Mão, Hospital de Clínicas, Universidade \\ Federal do Triângulo Mineiro, Uberaba, MG, Brasil \\ Rev Bras Ortop 2021;56(1):69-73.

\begin{abstract}
Endereço para correspondência Valdênia das Graças Nascimento Paiva, PhD, Ambulatório Maria da Glória, Serviço de Ortopedia e Traumatologia, Hospital de Clínicas da Universidade Federal do Triângulo Mineiro, Avenida Getúlio Guaritá, 130, Nossa Sra. da Abadia, Uberaba, MG, Brasil (e-mail: vallfmtm@yahoo.com.br).
\end{abstract}

\section{Resumo}

\section{Palavras-chave}

- eletroneuromiografia

- neuropatia compressiva

- síndrome do túnel do carpo

- ultrassonografia
Objetivo Verificar se existe associação entre os resultados da gravidade da eletroneuromiografia e a positividade da ultrassonografia no diagnóstico da síndrome do túnel do carpo. Métodos Sessenta e oito pacientes foram incluídos no estudo, sendo 61 mulheres e 7 homens, com média de idade de 54,4 anos. Os resultados da ultrassonografia (positivo ou negativo) foram cruzados com os resultados da eletroneuromiografia (leve, moderado ou grave) e verificada a existência de associação.

Resultados Cento e trinta e seis mãos com suspeita ou sintomas de síndrome do túnel do carpo foram avaliadas. O diagnóstico ultrassonográfico positivo foi observado em 72 mãos e negativo em 64; 123 mãos apresentaram eletroneuromiografia positiva para síndrome do túnel do carpo e 13 apresentaram resultado negativo. O grau grave da eletroneuromiografia foi prevalente.

Conclusão Houve associação estatisticamente significativa entre eletroneuromiografia e ultrassonografia $(p<0,05)$, sendo que a positividade da ultrassonografia foi maior para níveis mais graves de síndrome do túnel do carpo dados pela eletroneuromiografia.

\section{Abstract}

Objective To verify whether there is an association between the results of the severity in electroneuromyography and the positivity in ultrasound in the diagnosis of carpal tunnel syndrome.

Methods Sixty-eight patients were included in the study, 61 women and 7 men, with a mean age of 54.4 years. The ultrasound results (positive or negative) were crossed

\footnotetext{
* Trabalho feito no Serviço de Ortopedia e Traumatologia, Universidade Federal do Triângulo Mineiro, Uberaba, Minas Gerais, Brasil.
}

recebido

26 de Agosto de 2019

aceito

17 de Março de 2020

Publicado online

Outubro 9, 2020
DOI https://doi.org/

$10.1055 / \mathrm{s}-0040-1713390$.

ISSN $0102-3616$. (c) 2020. Sociedade Brasileira de Ortopedia e Traumatologia. All rights reserved.

This is an open access article published by Thieme under the terms of the Creative Commons Attribution-NonDerivative-NonCommercial-License, permitting copying and reproduction so long as the original work is given appropriate credit. Contents may not be used for commercial purposes, or adapted, remixed, transformed or built upon. (https://creativecommons.org/ licenses/by-nc-nd/4.0/)

Thieme Revinter Publicações Ltda., Rua do Matoso 170, Rio de Janeiro, RJ, CEP 20270-135, Brazil 


\section{Keywords}

- electro neuromyography

- compressive neuropathy

- carpal tunnel syndrome

- ultrasonography with the results of electroneuromyography (mild, moderate or severe), and the existence of association was verified.

Results One hundred and thirty-six hands with suspicion or symptoms of carpal tunnel syndrome were evaluated. Positive ultrasound diagnosis was observed in 72 hands and negative in 64; 123 hands presented positive electroneuromyography for carpal tunnel syndrome, and there were 13 negative results. The severe degree in electroneuromyography was prevalent.

Conclusion There was a statistically significant association between electroneuromyography and ultrasonography $(p<0.05)$, and ultrasound positivity was higher for more severe levels of carpal tunnel syndrome given by electroneuromyography.

\section{Introdução}

A síndrome do túnel do carpo (STC) é a neuropatia periférica mais comum decorrente da compressão do nervo mediano no túnel do carpo. ${ }^{1}$ O diagnóstico geralmente é feito por meio da história clínica e exame físico, ${ }^{2}$ enquanto a eletroneuromiografia (ENMG) auxilia na confirmação diagnóstica dos casos duvidosos e no estabelecimento da gravidade. ${ }^{3}$

A ultrassonografia (US) foi introduzida como ferramenta diagnóstica para STC no início dos anos $90,{ }^{4}$ sendo também utilizada para diagnóstico de algumas afecções musculoesqueléticas, como neuropatia do nervo ulnar e do nervo fibular. ${ }^{5-7}$ Um dos achados típicos da US relacionado à STC é o aumento da área de secção transversal do nervo mediano proximal ou distal ao local de compressão. ${ }^{8}$ Outros achados que podem ser identificados por esse exame são a diminuição da ecogenicidade e da mobilidade do nervo, aumento da vascularização, e variações anatômicas do nervo mediano, o que pode contribuir para o quadro clínico compressivo. A ENMG, por sua vez, é considerada o método diagnóstico de escolha para pessoas com suspeita de possuir neuropatias periféricas, fornecendo informações adicionais sobre a disfunção da mielina e a perda axonal. ${ }^{9}$

Estudos comparando diretamente a classificação da ENMG e a positividade da US para o diagnóstico da STC são escassos. Ademais, observamos em nossa prática clínica um grande número de pessoas com quadro clínico compatível com STC e resultado de exames divergentes entre si. Nosso estudo tem por objetivo verificar se existe associação entre os resultados da ENMG e a positividade da US no diagnóstico da STC.

\section{Casuística e métodos}

Foi realizado um estudo observacional, transversal, qualiquantitativo, com avaliação de 68 pacientes agendados de forma consecutiva no período de 4 meses em um ambulatório de cirurgia da mão de referência regional. Todos os procedimentos foram realizados conforme os padrões éticos determinados pelo Comitê de Ética em Pesquisa para pesquisa em seres humanos, e pela Declaração de Helsinque de 1964. O termo de consentimento livre e esclarecido foi obtido de todos os participantes, mediante assinatura de termo específico.

Os critérios de inclusão foram pessoas de ambos os gêneros, acima de 18 anos de idade, que apresentavam, na consulta inicial, ENMG de membros superiores e US de punho, ambos com hipótese diagnóstica de STC. Foram excluídas pessoas com outras neuropatias e/ou traumatismos prévios no punho, gestantes, pessoas com relatos de tenossinovites ou tumores no punho, pessoas com história conhecida de comorbidades sistêmicas não-controladas, e pessoas com relatos de atividades laborais atuais com movimentos repetitivos ou vibração de mãos.

Em relação à US, em nosso serviço, a equipe médica especializada em radiologia e diagnóstico por imagem define o ponto de corte seccional do nervo mediano sugestivo de anormalidade em $10 \mathrm{~mm}$ ou mais. A ENMG, por sua vez, é realizada pelo mesmo médico neurologista especializado em estudos eletroneuromiográficos, pertencente ao corpo clínico da instituição. A classificação da ENMG é assim padronizada: grau leve (alteração somente da condução sensitiva), grau moderado (alteração da condução sensitiva e motora), e grau grave (condução sensitiva e motora alteradas e sinais de denervação à eletromiografia de agulha).

As características qualitativas foram avaliadas em todos os pacientes e descritas por meio do uso de frequências absolutas e relativas. A variável quantitativa idade foi descrita por meio do uso de medidas resumo (média, desvio padrão, mediana, mínimo e máximo).

Foram atendidas 76 pessoas e excluídas 8 ( 1 com traumatismo prévio no punho, 2 com diabetes não controlado e 5 com cirurgias prévias no punho), totalizando 68 participantes incluídos neste estudo.

A - Tabela 1 apresenta as características clínicas das 68 pessoas que constituíram a nossa amostra. Em relação ao gênero, foram incluídas 61 mulheres $(89,7 \%)$ e 7 homens (10,3\%). A média e desvio padrão da idade foi 54,4 anos $\pm 10,1$. Quanto à situação laboral, 24 pessoas $(35,3 \%)$ informaram ser aposentadas ou do lar, enquanto $44(64,7 \%)$ estavam em atividade laborativa atual. Sessenta e cinco pessoas $(95,6 \%)$ relataram ser destras e $3(4,4 \%)$ canhotas. Em relação ao lado acometido pelos sintomas, 14 pessoas referiram sintomas somente à direita (20,6\%), 6 (8,8\%) 
Existe associação entre a eletroneuromiografia e a ultrassonografia no diagnóstico da STC? Paiva Filho et al. 71

Tabela 1 Descrição das características da amostra

\begin{tabular}{|l|l|}
\hline Variável & Descrição (N=68) \\
\hline Idade (anos) & \\
\hline média \pm DP & $54,4 \pm 10,1$ \\
\hline mediana (mín.; máx.) & $53,5(35 ; 76)$ \\
\hline Gênero, $\mathbf{n}$ (\%) & \\
\hline Feminino & $61(89,7)$ \\
\hline Masculino & $7(10,3)$ \\
\hline Profissão, $\mathbf{n}$ (\%) & \\
\hline Do lar/aposentado & $24(35,3)$ \\
\hline Em atividade laboral & $44(64,7)$ \\
\hline Dominância, $\mathbf{n}$ (\%) & \\
\hline Destro & $65(95,6)$ \\
\hline Canhoto & $3(4,4)$ \\
\hline Lado acometido, $\mathbf{n}(\%)$ & \\
\hline Direito & $14(20,6)$ \\
\hline Esquerdo & $6(8,8)$ \\
\hline Ambos & $48(70,6)$ \\
\hline Comorbidades, $\mathbf{n}$ (\%) & $24(35,3)$ \\
\hline Uma doença sistêmica & $16(23,5)$ \\
\hline Duas ou mais doenças sistêmicas & $28(41,2)$ \\
\hline Nenhuma doença relatada & \\
\hline
\end{tabular}

Abreviatura: DP, desvio padrão; n, número.

somente à esquerda, e 48 (70,6\%) informaram sintomatologia bilateral. Quando indagadas sobre comorbidades apresentadas, 28 pessoas $(41,2 \%)$ negaram possuir quaisquer comor- bidades, enquanto 24 (35,3\%) relataram apresentar 1 doença sistêmica e $16(23,5 \%) 2$ ou mais.

Os resultados da US (positivo ou negativo) foram cruzados com os resultados da ENMG (leve, moderado ou grave) e verificada a existência de associação com uso do teste quiquadrado. ${ }^{10}$ Foram calculados os coeficientes de concordância Kappa $^{11}$ para verificar o grau de concordância da US e da ENMG, bem como as medidas diagnósticas sensibilidade, especificidade, valor preditivo positivo (VPP) e valor preditivo negativo (VPN), para avaliar a predição de STC com uso da US.

Para realização das análises foi utilizado o software IBMSPSS for Windows versão 20.0 (IBM Corp., Armonk, NY, EUA) e para tabulação dos dados foi utilizado o software Microsoft Excel 2003 (Microsoft Corp., Redmond, WA, EUA). Os testes foram realizados com nível de significância de $5 \%$.

\section{Resultados}

Utilizando a mão como unidade amostral, a - Tabela 2 mostra o cruzamento entre US e ENMG, e o resultado dos testes estatístico e medidas diagnósticas. Das 136 mãos avaliadas, 123 apresentaram ENMG positiva para STC e 13 obtiveram resultado negativo para STC. Em relação à classificação da ENMG, 19 mãos foram consideradas com grau leve (15,5\%), 33 (26,8\%) grau moderado, e $71(57,7 \%)$ grau grave. Das 19 mãos com ENMG grau leve, 12 (63,2\%) apresentaram US negativa para STC e 7 (36,8\%) positiva. Em relação às 33 mãos com ENMG grau moderado, 19 (57,6\%) apresentaram US negativa para STC e $14(42,4 \%)$ positiva. Das 71 mãos com ENMG grau grave, 25 (35,2\%) apresentaram US negativa para STC e 46 (64,8\%) positiva. Por fim, das 13 mãos com ENMG negativa (ENMG normal), 8 (61,5\%) apresentaram US negativa para STC e $5(38,5 \%)$ positiva.

Tabela 2 Resultados do cruzamento entre ultrassonografia e eletroneuromiografia

\begin{tabular}{|c|c|c|c|c|c|c|c|c|c|}
\hline \multirow[t]{2}{*}{ Variável } & \multicolumn{2}{|c|}{ US para STC } & \multirow{2}{*}{$\begin{array}{l}\text { Total } \\
\mathrm{N}=136)\end{array}$} & \multirow[t]{2}{*}{$p$} & \multirow{2}{*}{$\frac{\text { Карра }}{\text { (IC 95\%) }}$} & \multirow{2}{*}{$\begin{array}{l}\text { Sens. } \\
\text { (IC 95\%) }\end{array}$} & \multirow{2}{*}{$\frac{\text { Espec. }}{\text { (IC 95\%) }}$} & \multirow{2}{*}{$\frac{\text { VPP }}{\text { (IC 95\%) }}$} & \multirow{2}{*}{$\frac{\text { VPN }}{\text { (IC 95\%) }}$} \\
\hline & $\begin{array}{l}\text { Negativo } \\
(\mathrm{N}=64)\end{array}$ & $\begin{array}{l}\text { Positivo } \\
(\mathrm{N}=72)\end{array}$ & & & & & & & \\
\hline ENMG & & & & 0,036 & & & & & \\
\hline Normal & $8(61,5)$ & $5(38,5)$ & 13 & & & & & & \\
\hline Leve & $12(63,2)$ & $7(36,8)$ & 19 & & & & & & \\
\hline Moderada & $19(57,6)$ & $14(42,4)$ & 33 & & & & & & \\
\hline Grave & $25(35,2)$ & $46(64,8)$ & 71 & & & & & & \\
\hline ENMG & & & & 0,271 & 0,058 & 54,5 & 61,5 & 93,1 & 12,5 \\
\hline Normal & $8(61,5)$ & $5(38,5)$ & 13 & & $(-0,046 ; 0,162)$ & $(45,2 ; 63,5)$ & $(31,6 ; 86,1)$ & $(84,5 ; 97,7)$ & $(5,6 ; 23,2)$ \\
\hline Alterada & $56(45,5)$ & $67(54,5)$ & 123 & & & & & & \\
\hline ENMG & & & & 0,045 & 0,150 & 57,7 & 62,5 & 83,3 & 31,3 \\
\hline Normal/leve & $20(62,5)$ & $12(37,5)$ & 32 & & $(0,003 ; 0,297)$ & $(47,6 ; 67,3)$ & $(43,7 ; 78,9)$ & $(72,7 ; 91,1)$ & $(20,2 ; 44,1)$ \\
\hline Moderada/grave & $44(42,3)$ & $60(57,7)$ & 104 & & & & & & \\
\hline ENMG & & & & 0,004 & 0,248 & 64,8 & 60,0 & 63,9 & 60,9 \\
\hline Normal/leve/moderada & $39(60)$ & $26(40)$ & 65 & & $(0,085 ; 0,411)$ & $(52,5 ; 75,8)$ & $(47,1 ; 72,0)$ & $(51,7 ; 74,9)$ & $(47,9 ; 72,9)$ \\
\hline Grave & $25(35,2)$ & $46(64,8)$ & 71 & & & & & & \\
\hline
\end{tabular}

Abreviaturas: ENMG, eletroneuromiografia; IC, intervalo de confiança; N, número; STC, síndrome do túnel do carpo; US, ultrassonografia; VPN, valor preditivo negativo; VPP, valor preditivo positivo; Sens., sensibilidade; Espec., especificidade.

Teste qui-quadrado. 


\section{Discussão}

Em estudos de base populacional, a prevalência da STC é maior em mulheres e aumenta com o envelhecimento, ${ }^{10}$ com estimativa de acometimento até três vezes mais que em homens. ${ }^{11}$ Isso pode ser explicado pelo fato de mulheres apresentarem o punho de tamanho menor ${ }^{12}$ e menor elasticidade do túnel do carpo, o que pode contribuir para menores complacência e acomodação das estruturas. ${ }^{13,14}$ É possível que mulheres tenham predisposição para túneis mais retificados, o que também pode contribuir para o desenvolvimento da STC. ${ }^{15}$ Com base nessas publicações, nosso trabalho está de acordo com a literatura, apresentando grande prevalência de acometimento da STC em mulheres.

Nos Estados Unidos, a STC é a terceira causa de afastamento laboral. ${ }^{16}$ Em nosso estudo, 35,3\% das pessoas com STC informaram ser aposentadas ou do lar, enquanto a maioria informou estar em atividade laborativa atual.

Alguns fatores de risco para STC incluem história pessoal e familiar, diabetes, obesidade, hipotireoidismo, gestação, e artrite reumatoide. ${ }^{17} \mathrm{Em}$ nossa casuística, mais da metade das pessoas apresentava pelo menos uma comorbidade sistêmica, dentre as quais as mais comumente citadas foram a hipertensão, diabetes, e hipotireoidismo.

A definição do ponto de corte seccional do nervo mediano pela US, sugestivo de STC, é controversa. A maioria dos artigos define o valor entre 8 e $14 \mathrm{~mm} .{ }^{18}$ Em nossa instituição, a equipe médica estipula o valor igual ou superior a $10 \mathrm{~mm}$ como sugestivo de anormalidade. Segundo Özçakar et al., ${ }^{19}$ a US apresenta a vantagem de ser de baixo custo, fácil utilização, não invasiva, portátil, disponível em grande parte dos serviços de saúde, e bem tolerada pelos pacientes. A mesma opinião também é compartilhada por Horng et al.; 20 entretanto, estes autores indicam o exame como complementação da ENMG. Em contrapartida, Mhoon et al. ${ }^{21}$ não encontram correlação significativa entre os parâmetros ultrassonográficos e a gravidade eletrofisiológica, e concluem que a US não é capaz de determinar a gravidade da STC. Para Abrishamchi et al.,22 a US pode ser complementar, mas não conclusiva, em relação à classificação da gravidade da STC. Para Fowler et al., ${ }^{8}$ a US e a ENMG têm acurácia diagnóstica semelhante aos testes clínicos, porém a ENMG pode diagnosticar outras etiologias de parestesia nas mãos além da STC, tais como radiculopatia cervical, síndrome do túnel cubital, e síndrome do pronador. ${ }^{23,24}$

Nosso estudo demonstrou que houve associação estatisticamente significativa entre ENMG e US $(p<0,05)$, sendo que a positividade da US foi maior para níveis mais graves de STC dados pela ENMG. Certamente este fato está relacionado à sensibilidade e ao alcance do método de US. Alterações do nervo mediano secundárias à compressão mais avançada são detectadas, enquanto as mais sutis ou iniciais não são. Cabe aqui a discussão se a decisão de indicação cirúrgica pode ser tomada com base apenas na US, já que somente os casos graves tiveram associação com a ENMG.

Apesar dessa associação, a US apresentou baixas concordâncias com a ENMG (Kappa <0,25), e as medidas diagnós- ticas foram, em sua maioria, baixas, para quaisquer que fossem os agrupamentos utilizados na gravidade da ENMG.

Concordamos com Fowler et al. ${ }^{8}$ quando estes afirmam que a comparação entre US e ENMG não pode ser considerada fidedigna pois não há um padrão de referência aceito. Ressaltamos, ainda, que ambos são exames que podem sofrer influência da experiência do examinador naquilo que se pretende investigar.

Acreditamos que decisões relativas ao diagnóstico da STC podem ser tomadas, inicialmente, com base na história clínica e no exame físico minucioso, direcionados àquilo que se pretende investigar.

\section{Conclusão}

Concluímos que, apesar da associação com significância estatística entre US e níveis mais graves da ENMG, os dois exames não apresentaram concordâncias significativas para o diagnóstico da STC.

Conflito de interesses

Os autores declaram não haver conflito de interesses.

\section{Referências}

1 Atroshi I, Gummesson C, Johnsson R, Ornstein E, Ranstam J, Rosén I. Prevalence of carpal tunnel syndrome in a general population. JAMA 1999;282(02):153-158

2 Wang WL, Buterbaugh K, Kadow TR, Goitz RJ, Fowler JR. A Prospective Comparison of Diagnostic Tools for the Diagnosis of Carpal Tunnel Syndrome. J Hand Surg Am 2018;43(09):833-836. e2

3 Sears ED, Swiatek PR, Hou H, Chung KC. Utilization of Preoperative Electrodiagnostic Studies for Carpal Tunnel Syndrome: An Analysis of National Practice Patterns. J Hand Surg Am 2016;41(06): 665-672.e1

4 Buchberger W, Schön G, Strasser K, Jungwirth W. High-resolution ultrasonography of the carpal tunnel. J Ultrasound Med 1991;10 (10):531-537

5 Kerasnoudis A, Tsivgoulis G. Nerve Ultrasound in Peripheral Neuropathies: A Review. J Neuroimaging 2015;25(04):528-538

6 Simon NG, Talbott J, Chin CT, Kliot M. Peripheral nerve imaging. Handb Clin Neurol 2016;136:811-826

7 Hobson-Webb LD, Padua L. Ultrasound of Focal Neuropathies. J Clin Neurophysiol 2016;33(02):94-102

8 Fowler JR, Cipolli W, Hanson T. A Comparison of Three Diagnostic Tests for Carpal Tunnel Syndrome Using Latent Class Analysis. J Bone Joint Surg Am 2015;97(23):1958-1961

9 Wee TC, Simon NG. Ultrasound elastography for the evaluation of peripheral nerves: A systematic review. Muscle Nerve 2019;60 (05):501-512

10 Kirkwood BR, Sterne JA. Essential medical statistics. 2nd ed. Massachusetts, USA: Blackwell Science; 2006

11 Fleiss JL. The design and analysis of clinical experiments. New York: Wiley; 1986

12 Mondelli M, Giannini F, Giacchi M. Carpal tunnel syndrome incidence in a general population. Neurology 2002;58(02): 289-294

13 Sassi SA, Giddins G. Gender differences in carpal tunnel relative cross-sectional area: a possible causative factor in idiopathic carpal tunnel syndrome. J Hand Surg Eur Vol 2016;41(06): 638-642

14 Lakshminarayanan K, Shah R, Li ZM. Sex-related differences in carpal arch morphology. PLoS One 2019;14(05):e0217425 
15 Bower JA, Stanisz GJ, Keir PJ. An MRI evaluation of carpal tunnel dimensions in healthy wrists: Implications for carpal tunnel syndrome. Clin Biomech (Bristol, Avon) 2006;21(08):816-825

16 Brett AW, Oliver ML, Agur AM, Edwards AM, Gordon KD. Quantification of the transverse carpal ligament elastic properties by sex and region. Clin Biomech (Bristol, Avon) 2014;29(06): 601-606

17 Cobb TK, Dalley BK, Posteraro RH, Lewis RC. Anatomy of the flexor retinaculum. J Hand Surg Am 1993;18(01):91-99

18 Barcenilla A, March LM, Chen JS, Sambrook PN. Carpal tunnel syndrome and its relationship to occupation: a meta-analysis. Rheumatology (Oxford) 2012;51(02):250-261

19 Harris-Adamson C, Eisen EA, Kapellusch J, et al. Biomechanical risk factors for carpal tunnel syndrome: a pooled study of 2474 workers. Occup Environ Med 2015;72(01):33-41

20 Tai TW, Wu CY, Su FC, Chern TC, Jou IM. Ultrasonography for diagnosing carpal tunnel syndrome: a meta-analysis of diagnostic test accuracy. Ultrasound Med Biol 2012;38(07):1121-1128
21 Özçakar L, Kara M, Chang KV, et al. Nineteen reasons why physiatrists should do musculoskeletal ultrasound: EURO-MUSCULUS/USPRM recommendations. Am J Phys Med Rehabil 2015; 94(06):e45-e49

22 Horng YS, Chang HC, Lin KE, Guo YL, Liu DH, Wang JD. Accuracy of ultrasonography and magnetic resonance imaging in diagnosing carpal tunnel syndrome using rest and grasp positions of the hands. J Hand Surg Am 2012;37(08):1591-1598

23 Mhoon JT, Juel VC, Hobson-Webb LD. Median nerve ultrasound as a screening tool in carpal tunnel syndrome: correlation of crosssectional area measures with electrodiagnostic abnormality. Muscle Nerve 2012;46(06):871-878

24 Abrishamchi F, Zaki B, Basiri K, Ghasemi M, Mohaghegh M. A comparison of the ultrasonographic median nerve crosssectional area at the wrist and the wrist-to-forearm ratio in carpal tunnel syndrome. J Res Med Sci 2014;19(12): $1113-1117$ 